\title{
Lyophilized phytosomal nanocarriers as platforms for enhanced diosmin delivery: optimization and ex vivo permeation
}

This article was published in the following Dove Press journal:

International Journal of Nanomedicine

2 July 2013

Number of times this article has been viewed

\author{
May S Freag \\ Yosra SR Elnaggar \\ Ossama Y Abdallah \\ Department of Pharmaceutics, \\ Faculty of Pharmacy, Alexandria \\ University, Alexandria, Egypt
}

Correspondence: Yosra SR Elnaggar Department of Pharmaceutics, Faculty of Pharmacy, Alexandria University, I Khartoum Square, Azarita, Messalla Post Office, PO Box 21521, Alexandria, Egypt Tel +20 II4 759 I065

Fax +2034873273

Email yosra_pharm@yahoo.com

\begin{abstract}
Diosmin (DSN) is an outstanding phlebotonic flavonoid with a tolerable potential for the treatment of colon and hepatocellular carcinoma. Being highly insoluble, DSN bioavailability suffers from high inter-subject variation due to variable degrees of permeation. This work endeavored to develop novel DSN loaded phytosomes in order to improve drug dissolution and intestinal permeability. Three preparation methods (solvent evaporation, salting out, and lyophilization) were compared. Nanocarrier optimization encompassed different soybean phospholipid (SPC) types, different solvents, and different DSN:SPC molar ratios (1:1, 1:2, and 1:4). In vitro appraisal encompassed differential scanning calorimetry, infrared spectroscopy, particle size, zeta potential, polydispersity index, transmission electron microscopy, drug content, and in vitro stability. Comparative dissolution studies were performed under sink versus non-sink conditions. Ex vivo intestinal permeation studies were performed on rats utilizing noneverted sac technique and high-performance liquid chromatography analysis. The results revealed lyophilization as the optimum preparation technique using SPC and solvent mixture (Dimethyl sulphoxide:t-butylalchol) in a 1:2 ratio. Complex formation was contended by differential scanning calorimetry and infrared data. Optimal lyophilized phytosomal nanocarriers (LPNs) exhibited the lowest particle size $(316 \mathrm{~nm})$, adequate zeta-potential $(-27 \mathrm{mV})$, and good in vitro stability. Well formed, discrete vesicles were revealed by transmission electron microscopy, drug content, and in vitro stability. Comparative dissolution studies were performed. LPNs demonstrated significant enhancement in DSN dissolution compared to crude drug, physical mixture, and generic and brand DSN products. Permeation studies revealed 80\% DSN permeated from LPNs via oxygenated rat intestine compared to non-detectable amounts from suspension. In this study, LPNs (99\% drug loading) could be successfully tailored for DSN with improved dissolution and permeation characteristics, which is promising for lowering the influence of exogenous factors and increasing drug delivery.
\end{abstract}

Keywords: flavonoids, phosphatidylcholine complex, nanomedicine

\section{Introduction}

Phytopharmaceuticals stand out as recent promising candidates for the treatment of chronic diseases and cancer. Fewer side effects and lower phytochemical costs from natural resources open new avenues for the treatment of various diseases and highlight the era of "back to nature". ${ }^{1}$ Flavonoids like quercetin, curcumin, luteolin, rutin, and diosmin (DSN) possess strong antioxidant activity ${ }^{2}$ as well as other interesting potential effects including anti-inflammatory, ${ }^{3}$ anti-cancer, ${ }^{1}$ and anti-ulcer ${ }^{4}$ activities. $\operatorname{DSN}(3,5$, 7-trihydroxy-4-methoxyflavone 7-rutinoside; Figure 1) is a flavone with outstanding therapeutic potential and a good safety profile with high tolerability. ${ }^{5}$ 


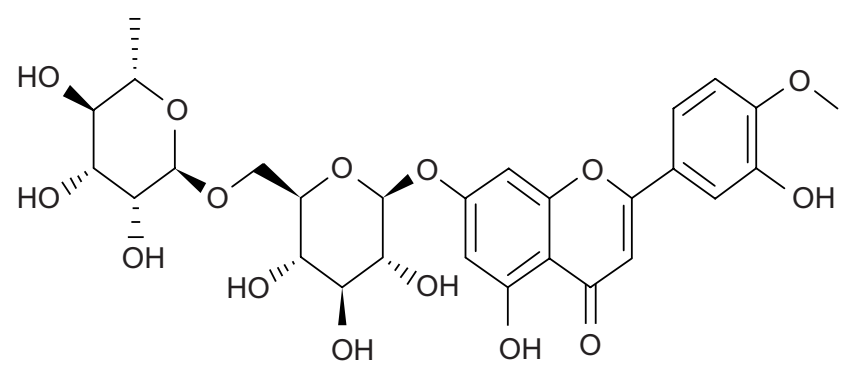

Figure I Chemical structure of diosmin.

DSN was first isolated in 1925 from Scrophularia nodosa, and first introduced as a therapeutic agent in 1969. It has been used for more than 30 years for its phlebotonic properties and as a vascular protector used for the treatment of hemorrhoids and venous leg ulcers. ${ }^{6}$ Furthermore, many researchers have considered DSN as an alternative treatment for hepatocellular, colon, and urinary bladder carcinogenesis with the advantages of lower costs and fewer adverse reactions compared to chemotherapy. ${ }^{1,7,8}$ DSN has also exhibited anti-inflammatory effects ${ }^{9}$ and reported anti-hyperglycemic activities. $^{10}$

Unfortunately, despite the wide therapeutic potential of flavonoids, their phenolic nature renders them polar but poorly water soluble, and scarce absorption is a reported consequence of these features. ${ }^{11}$ These aspects constitute a handicap against the widespread use of flavonoids in the pharmaceutical field. In particular, DSN is characterized by poor solubility in water and most organic solvents as well. Poor drug dissolution is responsible for its poor bioavailability and high inter-subject variation following oral administration. ${ }^{12}$ Low drug solubility results in reduced amounts of drug absorbed, paving the way for a high influence of exogenous factors, such as diet and dosage regimen. ${ }^{13}$ Therefore, a large standard dose of DSN (500 $\mathrm{mg}$ twice daily) is usually required for oral dosage regimens. ${ }^{13}$ A number of novel drug delivery systems have been investigated to overcome poor flavonoid dissolution, including quercetin liposomes, ${ }^{14}$ curcuminoids nanospheres ${ }^{15}$ and rutin microcapsules. ${ }^{16}$ Polymers, oils, or surfactants were utilized in these formulations to facilitate solubilization of poorly soluble flavonoids. Nevertheless, none of these delivery systems were formulated for DSN. This could be explained realizing poor solubilizing capacity of many surfactants and vehicles to the drug. ${ }^{12}$ One formulation attempt for enhancing DSN bioavailability that has been adopted is reducing particle size (PS) via the micronization process. Micronization improves the extent of DSN absorption, giving a pharmacokinetic explanation for the better clinical efficacy observed following the micronized formulation. ${ }^{13}$

DSN bioavailability can also be improved by formulating an appropriate drug delivery system, which can enhance the rate and the extent of drug absorption and permeation across the lipoid biomembrane. Natural soyabean phosphatidylcholines (SPC) have always presented as outstanding candidates for clinical applications including biocompatibility, biodegradability, metabolic activity, and low toxicity and cytotoxicity compared to their synthetic alternatives. ${ }^{17} \mathrm{In}$ this context, SPC based drug delivery systems are promising. Phytosomes (herbosomes) constitute complexes between a natural product and natural phospholipids, such as soy phospholipids. Such a complex is obtained by a reaction using stoichiometric amounts of SPC and the substrate in an appropriate solvent. The main phospholipid-substrate interaction is the formation of hydrogen bonds between the SPC polar head and the polar functionalities of the substrate. Such bonding is the main reported cause for the better physical stability profile of phytosomes compared to liposomes. ${ }^{18}$ Phytosomes have successfully improved the oral bioavailability of different flavonoids. In this context, Yanyu et al ${ }^{19}$ have developed silymarin phytosomes and investigated nanocarrier influence on drug pharmacokinetics in rats. Drug bioavailability was increased remarkably after oral administration of phytosomes due to an impressive improvement in the lipophilic properties of silybin-phospholipid complexes. Maiti et $\mathrm{al}^{20,21}$ developed the phytosomes of two flavonoids (curcumin and naringenin) in two different studies. The antioxidant activity of the complex was significantly higher than pure curcumin for all dose levels tested. Maiti et al developed the quercetin phospholipid phytosomal complex that showed better therapeutic efficacy than the molecule in a rat liver injury model. Nevertheless, no published research articles have thus far emerged investigating DSN loaded phyto vesicles and their influence on dissolution and intestinal permeation of the drug. It is anticipated that in the tailoring of such nanocarriers, obstacles, such as poor solubilizing capacity of different aqueous and oily vehicles to the drug, would render complex formation and its characterization more challenging.

Accordingly, this article has considered the genesis and appraisal of DSN loaded SPC phytosomes for enhanced drug dissolution and gastrointestinal permeation. The authors propose a new formulation for DSN described as lyophilized phytosomal nanocarriers (LPNs). The formulation constitutes selfnanovesicular pre-concentrates that will convert to a liquid state upon contact with gastrointestinal tract aqueous media. When 
treated with water, LPNs would assume a micellar shape while enjoying higher stability and better dosing privileges than the solid state. Optimization and full in vitro characterization of LPNs are carried out in this study. Ex vivo permeation studies on rats are also performed using the noneverted gut sac technique.

\section{Material and methods Material}

The SPC phospholipids including Lipoid ${ }^{\circledR}$ S100, Lipoid ${ }^{\circledR}$ S75, and hydrogenated SPC (Lipoid ${ }^{\circledR}$ S PC-3) were kind gifts from Lipoid Co, Ludwigshafen, Germany. DSN (purity 94\%) was purchased from Jianshi Yuantong Bioengineering Co, Ltd (Hubei, People's Republic of China). Dimethyl sulphoxide (DMSO) was purchased from (Oxford Laboratory, Maharashtra, India). T-butylalchol was purchased from Sigma (Sigma Aldrich Corporation, St Louis, MO, USA). Sodium dodecyl sulphate, n-hexane, and all other reagents and chemicals were of analytical grade.

\section{Preparation of phytosomes}

Three methods were compared for the development of DSN loaded SPC phytosomes, namely solvent evaporation, salting out, and lyophilization techniques. For all methods, Lipoid ${ }^{\circledR}$ S100 (Lipoid Co) was used as the SPC utilizing a DSN:SPC molar ratio of $1: 2$.

For the solvent evaporation method, DSN ( $0.1 \%$ weight/ volume) and SPC (Lipoid ${ }^{\circledR}$ S100; Lipoid Co) were suspended in a dioxane-methanol mixture $(7: 3)$, refluxed for 5 hours, then the solvent was evaporated under vacuum.

For the salting out method, DSN ( $0.1 \%$ weight/volume $)$ and SPC were dissolved in a mixture of DMSO, dehydrated ethanol, and chloroform $(2: 2: 3)$ to reach a final volume of $35 \mathrm{~mL}$. The solution was then stirred on a magnetic stirrer overnight then n-hexane $(75 \mathrm{~mL})$ was added until precipitation occurred.

For the lyophilization technique, DSN was completely dissolved in DMSO. The resulting DSN solution $(2.5 \%$ weight/volume) was added to the solution of SPC dissolved in t-butylalchol (1.5\% weight/volume) followed by stirring for 3 hours on a magnetic stirrer until complex formation. The complex was then isolated by lyophilization. The vials were frozen at $-80^{\circ} \mathrm{C}$ for 4 hours followed by placing them in a Cryodos-50 lyophilizer (Telstar Cryodos, Terrassa, Spain) with a condenser temperature of $-70^{\circ} \mathrm{C}$. Lyophilization was performed at a pressure of 40 mbar and a shelf temperature of $-40^{\circ} \mathrm{C}$ for 1-day followed by a secondary drying at $25^{\circ} \mathrm{C}$ for another day. After removing the samples from the freeze drier, the resultant DSN:SPC complex (yield 90.4\%, weight/weight) was placed in a desiccator over $\mathrm{P}_{2} \mathrm{O}_{5}$ at $4^{\circ} \mathrm{C}$ until testing. For the selected developing technique, the influence of variable formulation factors was assessed, including SPC type (Lipoid ${ }^{\circledR}$ S100, Lipoid ${ }^{\circledR}$ S75, and Lipoid ${ }^{\circledR}$ S PC-3), drug: phospholipid ratio $(1: 1,1: 2$, and 1:4) and co-solvent type (methanol, ethanol, chloroform, acetone, and TBA).

\section{Characterization of phytosomes Differential scanning calorimetry (DSC)}

Thermal behavior of the DSN:SPC complex was assessed using DSC analysis. The samples were sealed in an aluminum crimp cell and heated at a speed of $10^{\circ} \mathrm{C} /$ minute from 30 to $300^{\circ} \mathrm{C}$ in nitrogen atmosphere $(60 \mathrm{~mL} /$ minute $)$. Thermograms of DSN, SPC (Lipoid ${ }^{\circledR}$ S100), DSN:SPC complex (1:1; 1:2, and $1: 4)$, and physical mixture of DSN:SPC $(1: 1 ; 1: 2$, and 1:4) were obtained using the DSC 6 differential scanning calorimeter (PerkinElmer Inc, Shelton, CT, USA).

\section{Fourier transform infrared spectroscopy (FTIR)}

FTIR spectra were obtained using an FTIR spectrometer (PerkinElmer Inc). Samples were mixed with dry crystalline $\mathrm{KBr}$ in a ratio of 1:100 and pellets were prepared. A spectrum was collected for each sample within the wave number region 4000-500 $\mathrm{cm}^{-1}$. Samples assessed encompassed DSN, SPC (Lipoid $^{\circledR}$ S100), DSN:SPC complex (1:1, 1:2, and 1:4), and physical mixture of DSN:SPC (1:1, 1:2, and 1:4).

\section{Particle size, zeta-potential, and polydispersity index} The mean PS, polydispersity index (PDI), and zeta potential (ZP) of selected phytosomal formulation phytosomes were determined by dynamic light scattering (DLS) technique (Malvern Zeta Sizer, Malvern Instruments, Malvern, UK). The influence of cryoprotectant addition (mannitol 1\% weight/volume) on the PS, PDI, and ZP of selected phytosomal formulation was investigated as well. Samples were diluted with distilled water (1:10) before measurements. Assessed samples included DSN:SPC complex (1:1, 1:2, and 1:4) and DSN:SPC complex (1:2) with 1\% weight/ volume mannitol. Measurements were performed in triplicate.

\section{Drug content}

DSN content in phytosomes was determined spectrophotometrically. Approximately $5 \mathrm{mg}$ of the complex was dissolved in $25 \mathrm{~mL}$ of $1 \mathrm{M} \mathrm{NaOH}$. The resultant solution (3 mL/ $20 \mathrm{mg} \%$ ) was measured spectrophotometrically at $\lambda \max 266$ using SPC solution as blank. ${ }^{20}$

\section{Solubility studies}

Saturation solubility of DSN in water and different buffer systems was determined following a standard shake 
flask method. An excess quantity of DSN was added to $10 \mathrm{~mL}$ of solvent in a tightly capped glass vial. To achieve uniform mixing, samples were constantly agitated at $100 \mathrm{rpm}$ at $37^{\circ} \mathrm{C}$ for 24 hours in a reciprocating water bath (type 3047; Köttermann GmbH, Hänigsen, Germany). At the end of the 24-hour equilibrium at $37^{\circ} \mathrm{C}$, samples were centrifuged (Sigma Laboratory Refrigerated Centrifuge, Model 3K-30; Germany) and the supernatant was analyzed for drug content. Solubility was determined in water and in buffers with $\mathrm{pH}$ values ranging between 1.2 and 12 , namely $0.1 \mathrm{~N} \mathrm{HCL}$ ( $\mathrm{pH}$ 1.2), phosphate buffer ( $\mathrm{pH} 7.4)$, borate buffer $(\mathrm{pH} 9)$, borate buffer ( $\mathrm{pH}$ 10.5), and sodium orthophosphate buffer ( $\mathrm{pH}$ 12). Furthermore, influence of different concentrations of sodium dodecyl sulfate (SDS; 0.1, 0.5, and 1\% weight/ volume) on drug solubility in selected media was evaluated. SDS concentrations used for the phase solubility studies were $0.1,0.5,1 \%$ weight/volume in phosphate buffer $\mathrm{pH} 7.4$ and $0.1 \%, 0.5 \%$, and $1 \%$ weight/volume in borate buffer $\mathrm{pH} 10.5$. Saturation solubility samples were measured spectrophotometrically (UV-160 A; Shimadzu Co, Ltd, Kyoto, Japan) at a wavelength of $266 \mathrm{~nm}$ using the corresponding proper buffer as a blank.

\section{In vitro release study}

The dissolution studies were carried out using the USP XXIV dissolution rate apparatus II (Pharma Test Apparatebau AG, Hainburg, Germany) in two selected dissolution medias (borate buffer $\mathrm{pH} 9$ and sodium orthophosphate buffer $\mathrm{pH} 12)$. The dissolution medium $(500 \mathrm{~mL})$ was continuously stirred at $100 \mathrm{rpm}$ and $37^{\circ} \mathrm{C}$. Phytosomal formulations (equivalent to $55 \mathrm{mg} \mathrm{DSN}$ in sodium orthophosphate buffer and to $10 \mathrm{mg}$ DSN in borate buffer) were added to the stirred dissolution medium at time zero. ${ }^{19}$ Dissolution profiles of phytosomes with three different DSN:SPC ratios (1:1, 1:2, and 1:4) were compared to crude drug profiles. Furthermore, the dissolution patterns of selected phytosomal formulations were compared to physical mixtures of the same DSN:SPC ratio and to DSN generic and brand products currently available on the Egyptian market. Product tablets were ground and powder portions equivalent to aforementioned drug payloads in different buffers were weighed. Finally, the influence of cryoprotectant addition (mannitol 1\% weight/ volume) on dissolution behavior of selected was investigated as well. Comparison of all dissolution profiles was carried out in both release medias (borate buffer $\mathrm{pH} 9$ and sodium orthophosphate buffer $\mathrm{pH}$ 12). At different time intervals $(5,10,15,30,45$, and 60 minutes $), 3 \mathrm{~mL}$ samples were withdrawn and filtered using a Millipore filter (0.22 micrometer), followed by compensation with the same volume of fresh dissolution medium. Samples (in triplicates) were then measured spectrophotometrically at a wavelength of $266 \mathrm{~nm}$ using the corresponding proper buffer containing the corresponding SPC ratio as a blank.

\section{Transmission electron microscopy (TEM)}

Morphological examination of selected was carried out using TEM (model JEM-100S microscope; JOEL Ltd, Tokyo, Japan). Samples were diluted with distilled water (1:20) and sonicated for 3 minutes. A drop of the resultant phospholipid complex dispersions was placed onto a carboncoated copper grid, leaving a thin liquid film. The films on the grid were negatively stained by immediately adding a drop of $2 \%$ (weight/weight) ammonium molybdate in $2 \%$ (weight/volume) ammonium acetate buffer (pH 6.8). Excess staining solution was removed with a filter paper followed by air-dry. The stained films were then viewed and photographed using TEM.

\section{In vitro self-phytosomal stability}

The selected DSN phytosomes formulation was evaluated for in vitro stability in simulated gastric fluid (SGF; $\mathrm{pH} 1.2$ without enzymes) and simulated intestinal fluid (SIF; pH 7.4 without enzymes). Each milliliter of the formulation was added to $9 \mathrm{~mL}$ of SGF and SIF fluids and incubated for 2 hours and 6 hours, respectively. ${ }^{22}$ Samples were mildly agitated at $75 \mathrm{rpm}$ (at $37^{\circ} \mathrm{C}$ ) in a reciprocating water bath (type 3047; Köttermann). The samples were assessed for physicochemical properties (PS, ZP, and PDI) at time zero and after incubation using dynamic light scattering technique (Malvern Instruments). Experiments were carried out in triplicate.

\section{Ex vivo intestinal permeation studies}

Ex vivo permeation studies of DSN and its phytosomes were carried out using noneverted gut sac technique. ${ }^{23-25}$ A total number of 20 male Wistar rats (weighing 200-250 g) were obtained from Vacsera (Cairo, Egypt). Experiments were performed in accordance with the European Community guidelines for the use of experimental animals and were approved by the institutional ethics committee. The rats were housed in a temperature and humidity controlled room $\left(23^{\circ} \mathrm{C}\right.$, $55 \%$ air humidity) with free access to water and standard rat chow. The rats were acclimated for at least 5 days and fasted overnight but supplied with water ad libitum before the experiment. Animals were sacrificed by spinal dislocation and the small intestine was immediately removed after 
sacrifice by cutting across the upper end of the duodenum and the lower end of the ileum and manually stripping the mesentery. The small intestine was washed out carefully with cold normal oxygenated saline solution $(0.9 \%$, weight/ volume, $\mathrm{NaCl}$ ) using a syringe equipped with a blunt end. The clean intestinal tract was prepared into $8 \pm 0.2 \mathrm{~cm}$ long sacs having a diameter of $3.0 \pm 0.5 \mathrm{~mm}$.

Each sac was filled with $1 \mathrm{~mL}$ of selected LPN formulation (equivalent to $1 \mathrm{mg}$ of DSN) via a blunt needle, and the two sides of the intestine were tied tightly with a thread. Each noneverted intestinal sac was placed in a glass conical flask containing $10 \mathrm{~mL}$ of Ringer's solution (sodium chloride $[0.67 \%$, weight/volume]), potassium chloride $(0.034 \%$, weight/volume), magnesium sulphate $(0.059 \%$, weight/ volume), calcium chloride ( $0.011 \%$, weight/volume), sodium dihydrogen phosphate $(0.234 \%$, weight/volume $)$, and glucose $(0.18 \%$, weight/volume) in distilled water. In order to obtain sink conditions, a trace amount of $0.1 \mathrm{~N} \mathrm{NaOH}$ was added to adjust the medium to $\mathrm{pH}$ 9. The entire system was maintained at $37^{\circ} \mathrm{C}$ in a shaking water bath operated at 100 rpm and aerated with 5\% CO2 and 95\% O2 (10-15 bubbles/ minute) using a laboratory aerator. Samples were withdrawn from outside of the sac and the medium was totally replaced by fresh medium every 20 minutes for 2 hours. The samples were prepared for high performance liquid chromatography (HPLC) analysis by filtering through $0.45 \mu \mathrm{m}$ membrane filters. Studies were performed in triplicate and all data were statistically analyzed using the Student's $t$-test $(P<0.05){ }^{23}$

\section{High performance liquid chromatography (HPLC)}

A previously validated HPLC methodology developed by El-Shafae and El-Domiaty ${ }^{6}$ for DSN was utilized in this study with a slight modification. The HPLC instrument (Perkin Elmer series 200; PerkinElmer Inc) was equipped with a reversed-phase $\mathrm{C} 18$ column $(25 \mathrm{~cm} \times 4.6 \mathrm{~mm}$; particle size of $5 \mu \mathrm{m}$ ). The isocratic mobile phase (water:methanol; 60:40) was run at a flow rate of $1.5 \mathrm{~mL}$ per minute at room temperature and the column effluent was monitored using an ultraviolet (UV) detector set at $345 \mathrm{~nm}$. Samples $(20 \mu \mathrm{L})$ were automatically injected into the analytical column concurrently within the standard solution injections. The system was also equipped with a 600 Series Link Interface operated by Total Chrom chromatography data system software (v 6.2; PerkinElmer Inc). The calibration curve of peak area against DSN concentration was:

$$
Y=20.385 X+19.047
$$

under a concentration range of $5-50 \mu \mathrm{g} / \mathrm{mL}$. Retention time was $5.8 \pm 0.3$ minutes $\left(\mathrm{R}^{2}=0.993\right)$

\section{Statistical analysis}

Statistical analysis of the results was carried out using the Student's $t$-test $(P<0.05)$.

\section{Results and discussion \\ Preparation of preparatin of phytovesicles}

Three different preparation techniques were initially screened in this study for feasibility of complex formation; namely, solvent evaporation, salting out, and freeze drying. For preparation of DSN phytosomes, obtaining a clear drugphospholipid solution was mandatory. In our preliminary studies, we visually/qualitatively investigated the solubility of the poorly soluble drug in different organic solvents including methanol, ethanol, chloroform, acetone, methylene chloride, dimethyl formamide (DMF), and DMSO. It was observed that a clear DSN solution could be obtained using only DMF or DMSO, which is in accordance with reported solubility data. ${ }^{26}$ Nevertheless, DMSO has been proven to have a better safety profile (LD50 $7.920 \mathrm{~g} / \mathrm{kg}$; oral, mouse) compared to DMF (lethal dose $502.8 \mathrm{~g} \mathrm{~kg}^{-1}$; oral, rat), which is considered a possible carcinogen. ${ }^{27}$ The work by Bombardelli and Patri ${ }^{28}$ referred to solvent evaporation as a possible method for DSN:SPC complex formation. In accordance with this theory, the solvent evaporation technique was applied in this study using dioxane-methanol mixture (7:3) as a volatile solvent mixture. Nevertheless, no clear solution of DSN and SPC could be obtained in this solvent mixture, which hindered complex formation as is confirmed by our DSC study. Figure 2 shows DSC thermograms of SPC Lipoid ${ }^{\circledR}$ S100, DSN, physical mixture DSN:SPC (1:2), salting out method adduct, solvent evaporation method adduct, and freeze drying method adduct (DSN:SPC complex; 1:2). The inability of the solvent evaporation method to form a complex is confirmed in Figure 2 in which the adduct thermogram is identical to the physical mixture. It is noteworthy that DMSO could not be utilized in the solvent evaporation method owing to the high evaporation temperature of DMSO (boiling point $189^{\circ} \mathrm{C}$ ) that was anticipated to decompose phospholipids. ${ }^{29}$ On the other hand, in the salting out method, DSC analysis of the formed precipitate demonstrated that only pure DSN rather than the DSN:SPC complex was obtained upon addition of the complex non-solvent (n-hexane; Figure 2, thermogram D). The latter result could suggest the formation of a very weak complex that was decomposed upon addition of n-hexane, dissolving the phospholipid and leaving the drug precipitated. 


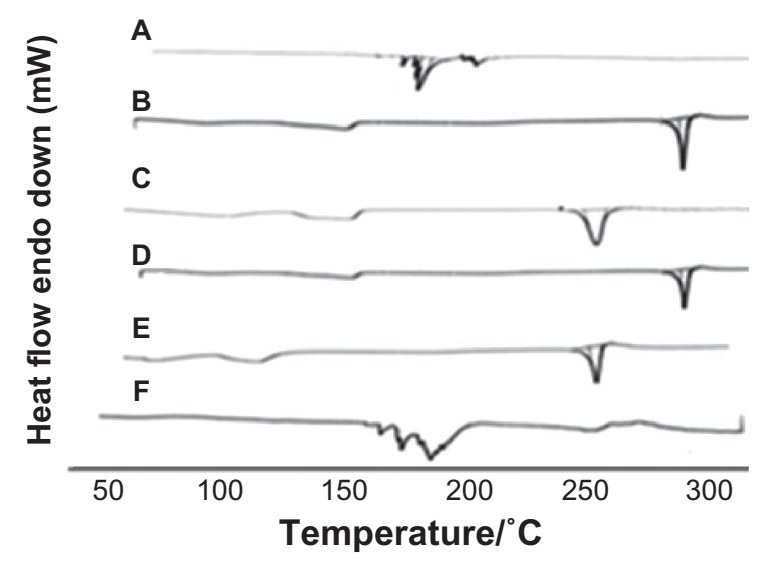

Figure 2 DSC thermograms of phospholipids SPC Lipoid ${ }^{\circledR}$ SI 00 (A), DSN (B), physical mixture DSN:SPC (I:2) (C), salting out method adduct (D), solvent evaporation method adduct $(\mathbf{E})$, and freeze drying method adduct (F; DSN:SPC ratio = I:2). Abbreviations: DSC, differential scanning calorimetry; DSN, diosmin; SPC, soyphosphatidylcholine.

As a third method, freeze drying was proposed for the preparation of LPNs. Such a technique would take the advantages of phytovesicle preparations in the dry form, avoiding the evaporation step and circumventing nonsolvent use. Furthermore, lyophilization was employed in this study as a one-step preparation technique rather than a second drying step for the prepared phytosomes as was utilized in other studies. ${ }^{17}$ DSC thermograms of lyophilized phytosomes will be discussed in detail in the DSC section (Figures 2 and 3).

\section{Selection of formulation factors}

For the chosen preparation technique (lyophilization), optimizing different formulation variables was then carried out. This encompassed selection of a proper co-solvent, proper SPC type, and optimum DSN:SPC ratio.

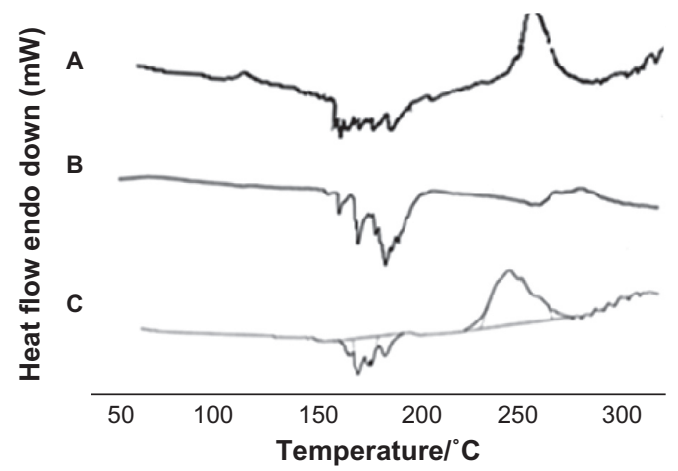

Figure 3 DSC thermograms of DSN:SPC complex in different ratios: $(1: 1 ; \mathbf{A})$, $(\mathrm{I}: 2 ; \mathbf{B})$, and $(1: 4 ; \mathbf{C})$.

Abbreviations: DSC, differential scanning calorimetry; DSN, diosmin; SPC, soyphosphatidylcholine.

\section{Co-solvent type}

After selection of DMSO as the main DSN solvent, the proper co-solvent for SPC was screened from methanol, ethanol, chloroform, acetone, and TBA. A co-solvent was required to dissolve SPC and at the same time to be mixed with DSN solution in DMSO without drug precipitation. Visual observation revealed that only TBA and methanol were able to dissolve SPC without affecting the physical stability of the DSN solution. However, the low freezing temperature of methanol hindered its use because sublimation during freeze drying is only possible when the solvent stays frozen. To obtain a lyophilization process of acceptable duration, the solvent should have a sufficiently high vapor pressure. DMSO has a high melting temperature $19^{\circ} \mathrm{C}$ but it has an extremely low vapor pressure $\left(0.08 \mathrm{kPa}\right.$ at $\left.25^{\circ} \mathrm{C}\right)$ so a slow drying process can be expected. Therefore, DMSO is not suitable as a solvent vehicle for freeze-drying. TBA possesses a high vapor pressure $\left(41.25 \mathrm{mmHg}\right.$ at $\left.25^{\circ} \mathrm{C}\right)$, a high melting point $\left(24^{\circ} \mathrm{C}\right)$ and has a low toxicity. ${ }^{30}$ Moreover, adding TBA to the lyophilization process was reported to produce large needle-shaped crystals with a high surface area and porosity that can facilitate sublimation. All these factors suggested TBA as an ideal freeze-drying medium that could be removed rapidly and completely by lyophilization. ${ }^{31}$ Therefore, TBA, which is miscible with DMSO in any proportion, was used as an organic co-solvent to solubilize SPC while the drug was dissolved in DMSO, then the mixed isotropic solution was lyophilized (DMSO:TBA 1:4). These results come in accordance with others who reported DMSO/TBA mixture as a freeze-drying medium for glibenclamide. ${ }^{32}$

\section{Phospholipid type}

After selection of the optimum formulation technique and solvent/co-solvent mixture, three different phospholipid types were screened for best formulation appraisal. In addition to Lipoid ${ }^{\circledR}$ S100, two other SPC were investigated, namely unsaturated Lipoid ${ }^{\circledR}$ S75 and the HSPC Lipoid ${ }^{\circledR}$ S PC-3. Only Lipoid $^{\circledR}$ S100 was soluble in the method co-solvent (TBA) leading to its choice as the SPC type for DSM phytosomes.

\section{Drug to phospholipid ratio}

A final formulation factor to be considered was the DSN:SPC ratio. The common drug: phospholipid ratio was fixed at 1:2 in the primary investigations while varying the abovementioned factors. In addition, two DSN:SPC ratios (1:1 and 1:4) were screened and characterized to give three phytosomal formulations (F1, F2, and F3) as depicted in Table 1. For the selected 
Table I Composition and characteristics of the diosmin-phospholipid lyophilized phytosomes

\begin{tabular}{lllcrc}
\hline Formula & $\begin{array}{l}\text { DSN/SPC } \\
\text { ratio }\end{array}$ & $\begin{array}{l}\text { Drug content } \\
(\% \mathbf{w} / \mathbf{w})\end{array}$ & $\begin{array}{l}\text { Particle size } \\
(\mathbf{n m})\end{array}$ & PDI & $\begin{array}{l}\text { Zeta potential } \\
(\mathbf{m V})\end{array}$ \\
\hline FI & I:I & $44.25 \pm 0.02$ & $537.5 \pm 3.66$ & $0.62 \pm 0.03$ & $-35.6 \pm 0.04$ \\
F2 & I:2 & $28.40 \pm 0.01$ & $316 \pm 2.45$ & $0.50 \pm 0.01$ & $-27.1 \pm 0.12$ \\
F3 & I:4 & $9.03 \pm 0.13$ & $350 \pm 3.01$ & $0.54 \pm 0.02$ & $-22.5 \pm 0.26$ \\
F4 & I:2+ I\% M & $22.12 \pm 0.03$ & $475 \pm 2.02$ & $0.41 \pm 0.02$ & $-16.3 \pm 0.14$ \\
\hline
\end{tabular}

Abbreviations: DSN, diosmin; F, formula; M, mannitol; PDI, polydispersity index; SPC, soy phosphatidylcholine; w, weight.

formulation, mannitol (1\% weight/volume) was added as a cryoprotectant during the lyophilization process. It is noteworthy that three nanocarrier formulations exhibited good self-phytosomal efficiency as confirmed by a low number of flask inversions required to prepare homogenous phytosomal dispersion. ${ }^{33,34}$ The three phytosomal formulations were then compared and characterized based on DSC analysis, infrared (IR) spectroscopy, PS, ZP measurement, PDI, and dissolution testing.

\section{DSC}

Thermal analysis is one of the crucial tools to characterize the solid state of matter, particularly in complex form. Consequently, DSC thermograms of SPC (A), DSN (B), DSN:SPC complex (1:2; C), and physical mixture (1:2; D) were obtained as previously depicted in Figure 2. The phospholipid thermogram demonstrated a sharp, pointed endothermal peak at $165.4^{\circ} \mathrm{C}$ that could be attributed to the transition from gel state to liquid crystal state. ${ }^{19,29}$ The DSN thermogram shows a sharp endothermal peak at $291.5^{\circ} \mathrm{C}$, corresponding to DSN melting. In the physical mixture thermogram, the endothermal peaks of both DSN and the phospholipid are still detectable but shifted towards lower temperatures $\left(261.4^{\circ} \mathrm{C}\right.$ and $140^{\circ} \mathrm{C}$, respectively). Maiti et $\mathrm{al}^{20}$ reported that when the temperature was increased, drugs would dissolve in the molten phospholipids and partly form phospholipid complexes, which could be explained by the theory of preparation by the melt out method. Nevertheless, the melt out method could not be exploited in this work owing to the high melting point of DSN $\left(291.5^{\circ} \mathrm{C}\right)$ that would lead to SPC decomposition. ${ }^{29}$ On the other hand, the DSC thermogram of all LPNs (Figure 3A-C) revealed the disappearance of the drug endothermal peak, with a broad endothermal peak appearing at $160^{\circ} \mathrm{C}$. This peak may be attributed to the formation of a new complex peak near the phospholipid peak or fusion of drug loaded phytosomes with SPC. These findings are in agreement with those obtained by $\mathrm{Xu}$ et $\mathrm{al}^{35}$ who reported disappearance of luteolin in SPC and explained complex formation by interactions including H-bonds or van der Waals forces.

\section{Fourier transform infrared spectroscopy (FTIR)}

Further investigation of complex formation was carried out using IR spectra spectroscopy. Figure 4 shows IR spectra of SPC (A), DSN (B), physical mixture (1:2; C), and F2 phytosomes (D). There was a slight difference between the physical mixture and the complex in the wavelength range from $1200 \mathrm{~cm}^{-1}$ to $960 \mathrm{~cm}^{-1}$ corresponding to the region of the SPC phosphate group. In addition, broadening of the characteristic phenolic $(-\mathrm{OH})$ band of DSN at $3500 \mathrm{~cm}^{-1}$ could be observed, which could be a sign of $\mathrm{H}$-bonding. The spectra of the physical mixture and the complex showed an additive effect of DSN and SPC, in which the characteristic absorption bands of phospholipid and DSN were still present at $1741 \mathrm{~cm}^{-1}$ and $3500 \mathrm{~cm}^{-1}$, respectively (stretching vibration mode). These observations suggest that some weak physical interactions between DSN $(-\mathrm{OH})$ and the phospholipid phosphate group took place during complex formation. These findings are also in agreement with those of $\mathrm{Xu}$ et $\mathrm{al}^{35}$ who reported that weak bonding between luteolin and phospholipid took place during the formation of the complex. IR spectra of the three LPN formulations (Figure 5) revealed no significant difference among all reflecting complex formation in all ratios.

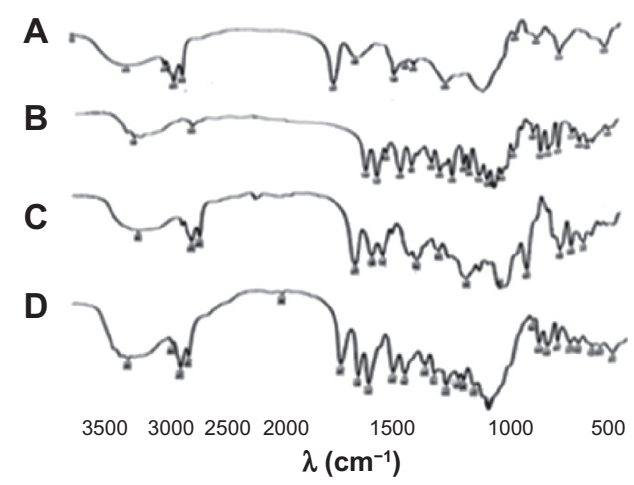

Figure 4 IR spectra of phospholipid SPC (A), DSN (B), DSN:SPC (I:2) complex (C), physical mixture of DSN:SPC (I:2; D).

Abbreviations: DSN, diosmin; IR, infrared; SPC, soyphosphatidylcholine. 


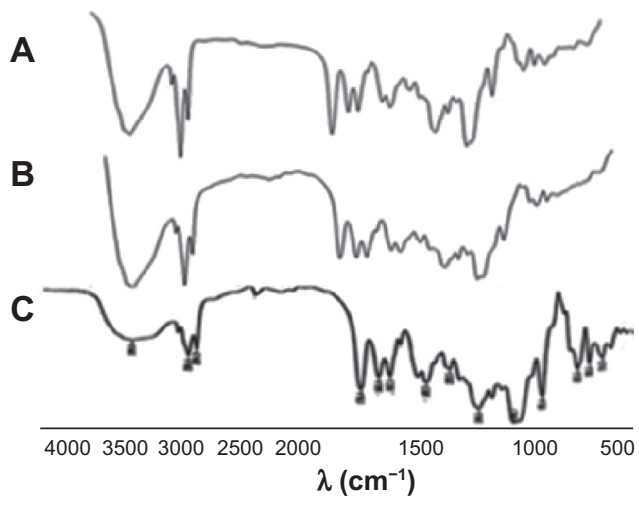

Figure 5 Infrared spectra of DSN:SPC complex with different ratios: (I:I; A), (I:2; B), and $(\mathrm{I}: 4 ; \mathbf{C})$.

Abbreviations: DSN, diosmin; SPC, soyphosphatidylcholine.

\section{Particle size, zeta-potential, and polydispersity index}

Physicochemical characterization of LPNs (F1, F2, F3, and F4) encompassed assessment of PS, ZP, and PDI as depicted in Table 1. Liquid phytosomes are anticipated to carry a negative charge ascribed to the SPC phosphate group in a water environment with a neutral $\mathrm{pH}$ value. ${ }^{17}$ The authors suggest that a ratio of DSN:SPC (1:1) in LPN formula F1 was not optimum for complete complexation of all DSN molecules. This was manifested by the highest PS $(537.5 \mathrm{~nm})$ and $\mathrm{ZP}(-35.6 \mathrm{mV})$ values reflecting possible free DSN and SPC due to weak bonding. On the other hand, the F2 formula (DSN:SPC, 1:2) revealed significant reduction in PS (316 nm) with an adequate $\mathrm{ZP}$ value $(-27.1 \mathrm{mV})$ and lower PDI ( 0.5$)$ compared to F1 (PDI $=0.62$ ). These findings suggested a DSN:SPC ratio of 1:2 as optimum for complex formation where all DSN molecules were physically bonded to the polar heads of SPC resulting in reduction of SPC negativity and phytovesicle particle size. Further increase in the DSN:SPC ratio beyond the proposed optimum ratio (1:4 in F3) resulted in significant increase in PS $(475 \mathrm{~nm}, \mathrm{PDI}=0.54)$ and a decrease in $\mathrm{ZP}(-22.5 \mathrm{mV})$. The latter effect might be attributed to aggregation of excess SPC molecules that have not undergone complexation with DSN leading to higher size and lower surface charge. The F4 formula (mannitol $1 \%$ ) exhibited higher particle size (475 $\mathrm{nm}$ ) compared to the corresponding F2 formula without cryoprotectant $(316 \mathrm{~nm})$. This might be attributed to the crystallization of mannitol and the formation of the eutectic composition with ice that can cause phase separation in the cryo-concentrated portion of the frozen phytosomes. Moreover, the growing crystals of water and mannitol may exert mechanical forces on the phytosomes leading to their fusion. ${ }^{36}$ Results revealed a significant reduction in $\mathrm{ZP}(-16.3 \mathrm{mV})$ compared to $\mathrm{F} 2(-27.1 \mathrm{mv})$. This effect could be explained via shielding of the negativity of
SPC by mannitol molecules which may result in lower ZP and decreased stability. The negative effects of cryoprotectants on PS and ZP of nanocarriers have been previously reported by other authors. ${ }^{36}$ No attempts have been adopted in this work to screen other drying agents.

It is noteworthy that trials to reduce formula PDI via extrusion or homogenization would not be appropriate for LPNs as such treatments are anticipated to decompose DSN:SPC complexes. At the same time, low PDI and high $\mathrm{ZP}$ values are more significant to liquid state nanocarriers that will remain during shelf time in the diluted state with high liability for aggregation. Liquefied nanocarriers would therefore usually require high ZP and low PDI values. On the other hand, self-nanocarrier pre-concentrates exemplified as LPNs carry the privilege of higher physical stability as they would exist in their diluted state for a maximum of 6 hours in the $\mathrm{GIT}^{34}$ thereby alleviating prerequisites of high ZP and low PDI. Therefore, maintenance of the initial physicochemical properties of LPNs would be more significant, which will be assessed as part of the self-phytosomal stability study.

\section{Solubility and in vitro release studies}

Saturation solubility of DSN in different buffer systems is demonstrated in Figure 6. Results revealed an increase in DSN solubility as the $\mathrm{pH}$ was shifted to the alkaline range. The highest solubilizing capacity of DSN ( $\mathrm{pka}=9.39-10.12$ ) was manifested in orthophosphate buffer $\mathrm{pH} 12$ (25 mg diosmin in $100 \mathrm{ml}$ orthophosphate buffer pH12). Incremental addition of SDS to selected media (phosphate buffer $\mathrm{pH} 7.4$, borate buffer $\mathrm{pH}$ 10.5) resulted in a small nonsignificant increase in saturation solubility when added at low concentration $0.1 \%$ and a slightly higher increase in saturation solubility at higher concentrations $0.5 \%$ and $1 \%$. Nevertheless, high SDS concentrations imparted turbidity to drug solutions that would cause interference during DSN

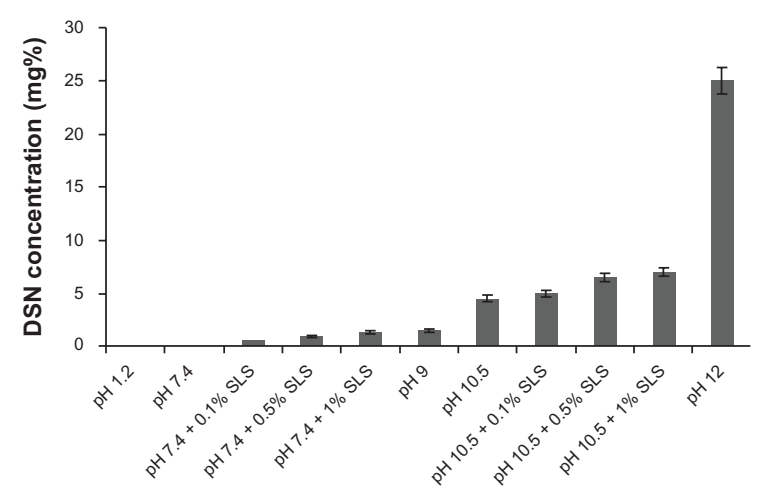

Figure 6 Saturation solubility of DSN in different buffer systems. Abbreviations: DSN, diosmin; SLS, sodium laurylsulfate. 
spectrophotometric analysis. Therefore, continuing with SDS as a release enhancer was discouraged.

Due to its poor solubility, development of an appropriate dissolution test for DSN constitutes a challenging task. Garner et $\mathrm{al}^{13}$ reported the necessary presence of high quantities of sodium hydroxide to achieve sink conditions to perform a suitable dissolution profile of DSN. In the current study, sodium orthophosphate buffer ( $\mathrm{pH} 12)$ was the medium of the highest solubilization capacity of DSN (Figure 6) and the only buffer system that was so far reported for DSN dissolution. ${ }^{37}$ Consequently, it was selected as the dissolution medium achieving sink conditions. On the other hand, another reported point of view adopted higher suitability of non-sink conditions to reflect in vivo conditions of poorly soluble drugs. Ghazal et a ${ }^{38}$ reported that sink conditions may not prevail in the GIT for itraconazole as a poorly soluble drug and performed a comparative dissolution study in non-sink media. The initial linear portion of each curve representing intrinsic solubility was utilized for comparative purposes. Accordingly, another non-sink dissolution medium for DSN (borate buffer $\mathrm{pH}=9$ ) was utilized in this work to investigate versus sink conditions of orthophosphate buffer. Percentage of dissolved drug after 15 minutes (PD15) was measured for all samples studied.

For orthophosphate and borate media, dissolution profiles of DSN crude drug was compared to different LPN formulations F1, F2, and F3. Dissolution profiles were depicted in Figures 7 and 8. Results revealed significant enhancement of DSN release in both orthophosphate (Figure 7) and borate buffer (Figure 8) from all LPN formulations compared to plain drug. So far, LPNs characterization revealed complex formation for all formulations $\mathrm{F} 1, \mathrm{~F} 2$, and F3 as indicated by DSC and IR data. Whereas F2 formula exhibited the lowest PS, PDI, and adequate ZP parallel to significant dissolution enhancement. Therefore, phytosomal formula F2 with a DSN:SPC ratio of 1:2

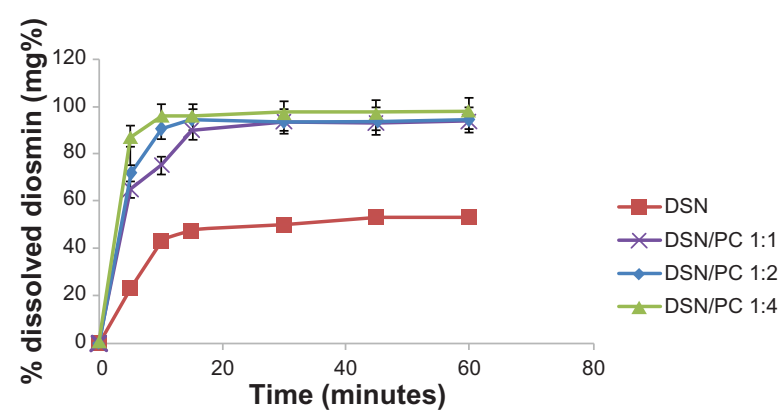

Figure 7 Dissolution behavior of DSN:SPC (1:1, I:2, and 1:4) complex and crude DSN in sodium orthophosphate buffer at $\mathrm{pH} 12$.

Abbreviations: DSN, diosmin; PC, phosphatidylcholine; SPC, soyphosphatidylcholine.

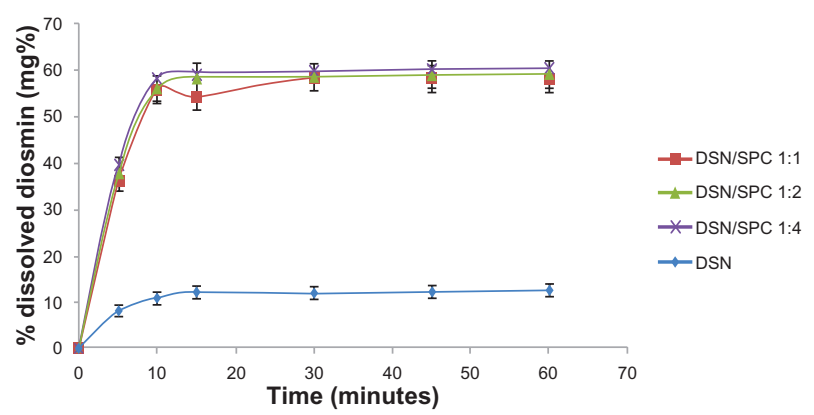

Figure 8 Dissolution behavior of DSN:SPC (I:I, I:2, and I:4) complex and crude DSN in borate buffer at $\mathrm{pH} 9$.

Abbreviations: DSN, diosmin; SPC, soyphosphatidylcholine.

was selected as the optimized formulation for further investigations.

The second part of the dissolution studies concerned comparing essential dissolution profiles. Drug release from the selected formula (F2) was compared to generic product and brand product with the same drug payload (11 $\mathrm{mg}$ diosmin in $100 \mathrm{ml}$ buffer) in orthophosphate buffer (Figure 9) and drug payload ( $2 \mathrm{mg}$ diosmin in $100 \mathrm{ml}$ buffer) in borate buffer (Figure 10). Furthermore, the dissolution profile of F2 was compared to the physical mixture of the same DSN:SPC ratio $(1: 2)$ and to the corresponding formula with mannitol (1\% weight/volume) as a cryoprotectant (F4) in both dissolution medias. Results demonstrated enhanced DSN dissolution when physically mixed with SPC showing a PD15 of $64.9 \%$ in buffer $\mathrm{pH} 12$ and a PD15 of $15.5 \%$ in buffer $\mathrm{pH} 9$ compared to plain drug (PD15 $=47.6 \%$ and $12.4 \%$, respectively). This could be attributed to the improved drug wettability due to the presence of SPC that reduces the interfacial tension between the water insoluble drug particles and the dissolution medium. Such a slow dissolution rate of DSN in both medias could be attributed to DSN lyophobicity and consequent agglomeration. On the other hand, LPN formulations F1, F2, and F3

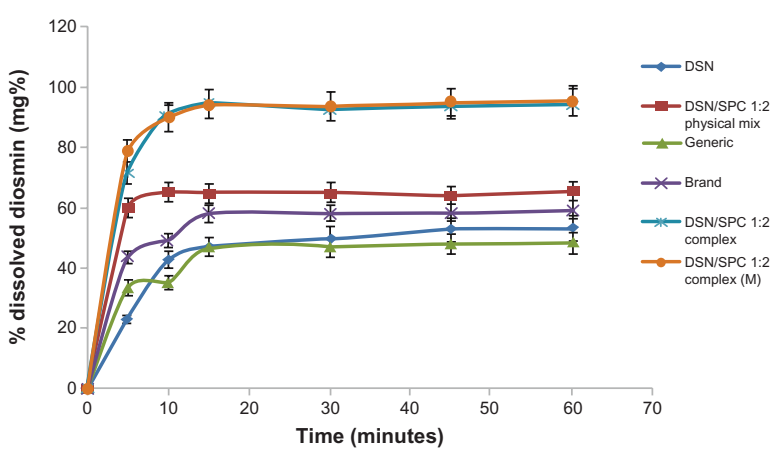

Figure 9 Dissolution profiles of crude DSN, DSN:SPC (1:2) complex, DSN:SPC $(I: 2)$ physical mixture, DSN:SPC (I:2) complex + I\% mannitol, and generic and brand product in sodium orthophosphate buffer at $\mathrm{pH} 12$.

Abbreviations: DSN, diosmin; M, mannitol; SPC, soyphosphatidylcholine. 


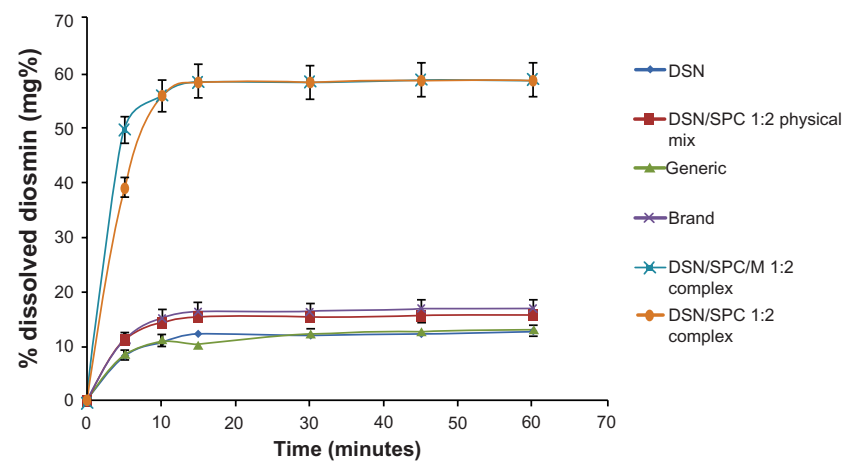

Figure 10 Dissolution profiles of crude DSN, DSN:SPC complex (1:2), DSN:SPC physical mixture (I:2), DSN:SPC (I:2) complex + I\% mannitol, generic and brand product in borate buffer at $\mathrm{pH} 9$.

Abbreviations: DSN, diosmin; SPC, soyphosphatidylcholine; M, mannitol.

demonstrated a PD15 of $90 \%, 94.6 \%$, and $96.3 \%$, respectively in $\mathrm{pH} 12$, and a PD15 of 54.3\%, 58.6\%, and $59.3 \%$ in $\mathrm{pH} 9$ buffers, respectively. In addition, Figures 9 and 10 reveal a significant increase in DSN dissolution rate from LPNs compared to both brand and generic products currently available on the Egyptian market. Brand and generic products showed a PD15 of $58 \%$ and $47 \%$, respectively, compared to $94.6 \%$ for $\mathrm{F} 2$ in $\mathrm{pH} 12$, brand and generic products exhibited a PD15 of $16.5 \%$ and $10.6 \%$, respectively, compared to $59.3 \%$ for $\mathrm{F} 2$ in $\mathrm{pH}$ 9. Results suggested against any significant effect of the drying agent addition on F2 dissolution profiles either in sodium orthophosphate buffer (PD15 of 94.4\%) or in borate buffer (PD15 of 58.9\%).

The dissolution of solid dosage forms is a complex operation influenced by a number of factors. Differences in surface area, surface energies, PS, and wetting properties may all play a role in affecting the dissolution rate of powder. ${ }^{31}$ Such significant enhancement in dissolution of LPNs compared to pure drug, and generic and brand products could be ascribed mainly to the amphiphilic nature of the complex. Soyabean phospholipids, being an amphiphilic surfactant, are anticipated to increase DSN solubility through the action of wetting and dispersion. Furthermore, the nano size of the phytovesicles could be another factor for dissolution enhancement of DSN. This explanation could contend with PS results demonstrating a nano-metric range for all complex ratios reflecting high specific surface area compared to plain drug (Table 1).

\section{Transmission electron microscopy}

The TEM (Figure 11) of the selected LPN formula (F2) revealed well formed, discrete vesicles. For the phospholipids complex, the drugs are combined with phospholipids by the polar part of the phospholipids. When swirled in

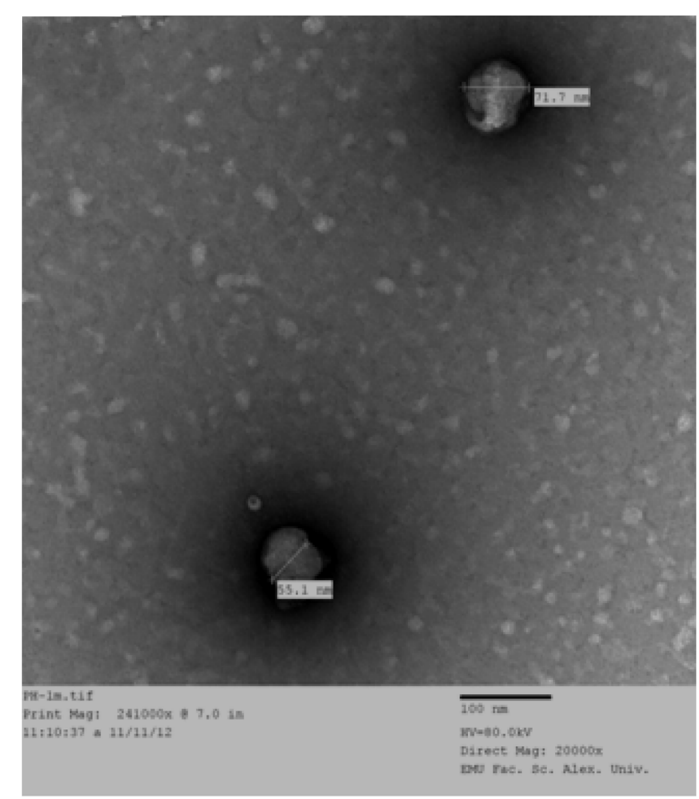

Figure II Transmission electron microscopic photographs of F2 phytosomes with 20 -fold dilution in distilled water $(\times 20,000)$.

Abbreviation: F, formula.

distilled water, LPNs were arranged in order and formed phytovesicular structures with no morphological signs of aggregation or decomposition.

\section{In vitro self-phytosomal stability}

In order to predict the physical stability of LPNs after ingestion into the GIT, monitoring of physicochemical properties (PS, PDI, and ZP) and physical changes (aggregation and precipitation) of $\mathrm{F} 2$ has been carried out for the postulated duration in GIT in vivo. Results of the in vitro phytosomal stability study are summarized in Table 2. Stability of F2 after 2 hours in SGF (pH 1.2) revealed reduction in the PS upon incubation with the SGF ( $\mathrm{pH} 1.2$ ) from $201.5 \pm 2.12 \mathrm{~nm}$ to $122 \pm 1.22 \mathrm{~nm}$. Insignificant changes in initial PDI $(0.91 \pm 0.02)$ and $\mathrm{ZP}(+21.1 \mathrm{mV})$ of LPNs were evident after 2 hours (PDI $1 \pm 0.01, \mathrm{ZP}+24.10 \pm 1.52 \mathrm{mV}$ ). Formula stability in SIF ( $\mathrm{pH}$ 7.4) revealed insignificant changes in

Table 2 In vitro self-phytosomal stability of selected LPN formulation (F2) in SGF and SIF without enzymes (data expressed as mean of triplicates \pm standard deviation)

\begin{tabular}{lrrr}
\hline Time interval & \multicolumn{1}{c}{ PS $(\mathbf{n m})$} & \multicolumn{1}{c}{ PDI } & \multicolumn{1}{c}{ ZP $(\mathbf{m V})$} \\
\hline Zero time (SGF) & $201.5 \pm 2.12 \mathrm{~nm}$ & $0.91 \pm 0.02$ & $+21.1 \pm 1.13$ \\
2-hour (SGF) & $122 \pm 1.22 \mathrm{~nm}$ & $\mathrm{I} \pm 0.01$ & $+24.10 \pm 1.52$ \\
Zero time (SIF) & $477.70 \pm 3.11 \mathrm{~nm}$ & $0.615 \pm 0.02$ & $-5.1 \pm 0.13$ \\
6-hour (SIF) & $438.40 \pm 2.25 \mathrm{~nm}$ & $0.63 \pm 0.01$ & $-4.90 \pm 0.12$ \\
\hline
\end{tabular}

Abbreviations: LPN, lyophilized phytosomal nanocarriers; PDI, polydispersity index; PS, particle size; SGF, simulated gastric fluids; SIF, simulated intestinal fluids; ZP, zetapotential. 
initial PS (477.70 $\pm 3.11 \mathrm{~nm})$, PDI $(0.615 \pm 0.02)$, and ZP $(-5.1 \pm 0.13 \mathrm{mV})$ compared to corresponding values after 6 hours (PS $=438.40 \pm 2.25 \mathrm{~nm}$; PDI $=0.63 \pm 0.01$; and $\mathrm{ZP}=-4.90 \pm 0.12 \mathrm{mV})$. No signs of aggregation or precipitation have been observed either in SGF or SIF media.

Results inferred that LPNs could maintain their initial physicochemical properties and homogenous dispersion stability after incubation in different $\mathrm{pH}$ media and a challenging dilution fold (1:9), reflecting good physical stability of the nanocarriers. Thorough investigation of the results infers that LPNs carry a positive charge in acidic medium $(24 \mathrm{mV})$, a negative charge in the higher $\mathrm{pH}$ of water $(-27 \mathrm{mV}$; Table 1), and a lower negative charge in phosphate buffer $\mathrm{pH} 7.4(-6 \mathrm{mV})$. These changes in the vesicular surface charge might be explained based on the dual charge of soya phosphatidylcholine (intrinsic SPC pka $=0.8$, negative phosphate group and positive choline molecule). Acidic medium is supposed to neutralize phosphate oxygen negativity allowing positive charge to predominate. On the other hand, higher $\mathrm{pH}$ (water and SIF) is anticipated to neutralize choline positive charge leading to negatively charged vesicles. Lower negative value of SIF compared to water may be attributed to common ion effect of the $\mathrm{PO}_{4}$ group on SPC leading to negative charge suppression. Such a low ZP value could maintain initial physical stability of the self-phytosomal nanocarriers for the required time in GIT without any precipitation or particle aggregation. The results obtained from $\mathrm{ZP}$ analysis suggest that the nanocarrier pre-concentrates do not require high repulsion forces for stabilization due to dispensable need for the liquefied state on shelf. ${ }^{33}$ An observed lower PS of LPNs in acidic medium may be ascribed to discouraged ionization of the phosphate group in water leading to vesicular shrinkage with lower PS and PDI as a consequence.

\section{Ex vivo intestinal permeation studies}

In vitro absorption models are commonly used to investigate transport mechanisms, classify permeability, and predict in vivo drug absorption in humans for reduced labor and experimental costs compared to in vivo studies. ${ }^{39}$ Among others, everted and noneverted intestinal sac models in rats have commonly been employed to study drug absorption. ${ }^{40-42}$ Genty et $\mathrm{al}^{43}$ have compared permeability values of some actively and passively transported molecules through both everted and non-everted sacs. They reported that the permeability was higher for actively transported molecules when the sacs were everted. Meanwhile, the permeability of passive absorption drug remained the same whether the sacs were everted or not. These results suggested that passive permeability of actively transported molecules can be determined through non-everted rat gut sacs. ${ }^{43}$ The non-everted sac model utilized in this work exhibited reported advantages over the everted sac model, including simplicity, need for less test sample, and amenability for successive collection of serosal samples with less intestinal morphological changes. ${ }^{44}$ The well-known limitations of the everted gut sac model are morphological damages to intestinal tissue while everting, ${ }^{45}$ presence of muscularis mucosa, and small closed serosal compartment. ${ }^{46}$ Oxygenated tissue culture media (TC 199) and specific preparation techniques ensured tissue viability of intestinal sacs for up to 2 hours. ${ }^{47}$

Intestinal permeation profiles for the selected LPN formula (F2) and crude DSN suspension are depicted in Figure 12. The study was carried out in Ringer's solution with a $\mathrm{pH}$ adjusted to nine using $0.1 \mathrm{~N} \mathrm{NaOH}$ to achieve near sink conditions ( 1.5 folds sink). These conditions were not harmful for the intestinal sac as previously reported by Ruan et al. ${ }^{23}$ Results demonstrate that non-detectable amounts of DSN could be found in the permeation flask of crude drug all over the study time. On the other hand, the phytosomal formula F2 exhibited $80.10 \%$ DSN permeated after 120 minutes. Such significant enhancement in permeation could be ascribed to the phospholipid molecular structure including a water-soluble head and two fat-soluble tails. Because of this dual solubility, the phospholipid acts as an effective emulsifier. By combining the emulsifying action of the SPC with the nanometric size, phytosomes could provide dramatically enhanced permeation and consequently

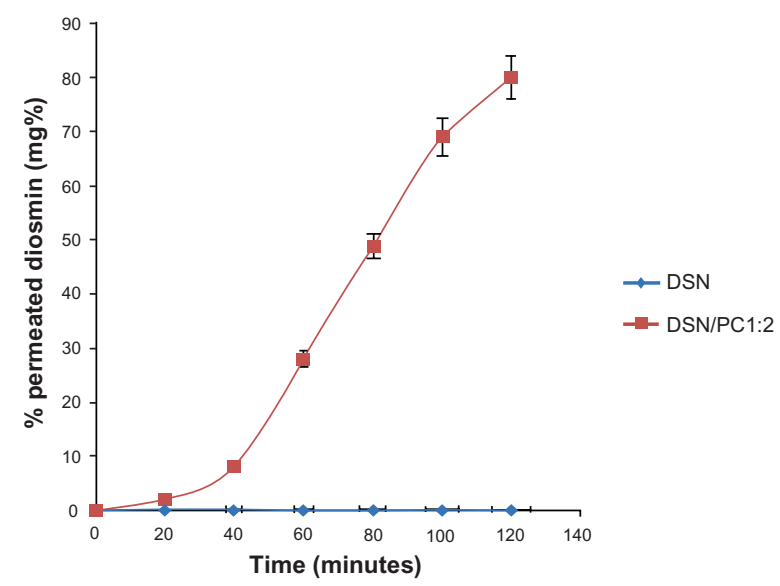

Figure 12 Cumulative permeation of F2 phytosomes and crude DSN through noneverted rat intestine in Ringer's buffer medium $\left(\mathrm{pH}\right.$ 9) at $37^{\circ} \mathrm{C}$.

Abbreviations: DSN, diosmin; F, formula; PC, phosphatidylcholine. 
bioavailability for lipid soluble drugs explained by faster and improved absorption in the intestinal tract. ${ }^{18}$

\section{Conclusion}

The current work adopted development and in vitro appraisal of a new nanocarrier formulation of a DSN:SPC complex. LPNs could be successfully developed via the freeze drying technique. The amphiphilic phytovesicles could be tailored for the drug with $99.12 \% \pm 0.09 \%$ drug loading, spherical shape, and nanometric range. Phytovesicles exhibited good physicochemical stability in different $\mathrm{pH}$ media and spherical discrete particles. Two sink and non-sink dissolution conditions have been proposed for DSN. Results of dissolution studies in both conditions contended the ability of lyophilized phytosomal nanocarriers to enhance dissolution rate of the drug compared to its crude form and marketed products. Furthermore, LPNs could significantly improve DSN intestinal permeation via non-everted rat sacs. DSN loaded nanovesicles tailored in this work exhibited promising in vitro and ex vivo properties paving the way for better delivery characteristics of such a poorly soluble and permeable drug.

\section{Disclosure}

The authors report no conflicts of interest in this work.

\section{References}

1. Dung TD, Day CH, Binh TV, et al. PP2A mediates diosmin p53 activation to block HA22T cell proliferation and tumor growth in xenografted nude mice through PI3K-Akt-MDM2 signaling suppression. Food Chem Toxicol. 2012;50(5):1802-1810.

2. Kandaswami C, Middleton E. Free radical scavenging and antioxidant activity of plant flavonoids. Adv Exp Med Biol. 1994;366:351-376.

3. Di Perri T, Auteri A. Action of S 5682 on the complement system. In vitro and in vivo study. Int Angiol. 1988;7(Suppl 2):11-15.

4. Izzo AA, Carlo GD, Mascolo N, Capasso F, Autore G. Antiulcer effect of flavonoids. Role of endogenous PAF. Phytother Res. 1994;8: 179-181.

5. Hitzenberger G. [Therapeutic effectiveness of flavonoids illustrated by daflon $500 \mathrm{mg}$.] Wien Med Wochenschr. 1997;147(18):409-412. German.

6. El-Shafae AM, El-Domiaty MM. Improved LC methods for the determination of diosmin and/or hesperidin in plant extracts and pharmaceutical formulations. J Pharm Biomed Anal. 2001;26(4):539-545.

7. Tanaka T, Makita H, Kawabata K, et al. Chemoprevention of azoxymethane-induced rat colon carcinogenesis by the naturally occurring flavonoids, diosmin and hesperidin. Carcinogenesis. 1997;18(5): 957-965.

8. Yang M, Tanaka T, Hirose Y, Deguchi T, Mori H, Kawada Y. Chemopreventive effects of diosmin and hesperidin on N-butyl-N-(4hydroxybutyl)nitrosamine-induced urinary-bladder carcinogenesis in male ICR mice. Int J Cancer. 1997;73(5):719-724.

9. Lonchampt M, Guardiola B, Sicot N, Bertrand M, Perdrix L, Duhault J. Protective effect of a purified flavonoid fraction against reactive oxygen radicals. In vivo and in vitro study. Arzneimittelforschung. 1989;39(8): 882-885.
10. Pari L, Srinivasan S. Antihyperglycemic effect of diosmin on hepatic key enzymes of carbohydrate metabolism in streptozotocin-nicotinamideinduced diabetic rats. Biomed Pharmacother. 2010;64(7):477-481.

11. Havsteen BH. The biochemistry and medical significance of the flavonoids. Pharmacol Ther. 2002;96(2-3):67-202.

12. Serra H, Mendes T, Bronze MR, Simplício AL. Prediction of intestinal absorption and metabolism of pharmacologically active flavones and flavanones. Bioorg Med Chem. 2008;16(7):4009-4018.

13. Garner RC, Garner JV, Gregory S, Whattam M, Calam A, Leong D. Comparison of the absorption of micronized (Daflon $500 \mathrm{mg}$ ) and nonmicronized 14C-diosmin tablets after oral administration to healthy volunteers by accelerator mass spectrometry and liquid scintillation counting. J Pharm Sci. 2002;91(1):32-40.

14. Priprem A, Watanatorn J, Sutthiparinyanont S, Phachonpai W, Muchimapura S. Anxiety and cognitive effects of quercetin liposomes in rats. Nanomedicine. 2008;4(1):70-78.

15. Mukerjee A, Vishwanatha JK. Formulation, characterization and evaluation of curcumin-loaded PLGA nanospheres for cancer therapy. Anticancer Res. 2009;29(10):3867-3875.

16. Xiao, Zhang YH, Xu JC, Jin XH. Preparation of floating rutin-alginatechitosan microcapsule. Chin Trad Herb Drugs. 2008;2:209-212.

17. Hou Z, Li Y, Huang Y, et al. Phytosomes loaded with mitomycin C-soybean phosphatidylcholine complex developed for drug delivery. Mol Pharm. 2013;10(1):90-101.

18. Vinod KR, Sandhya S, Chandrashekar J, et al. Review on genesis and characterization of phytosomes, Review article, IJPSRR. 2010;4:69-75.

19. Yanyu X, Yunmei S, Zhipeng C, Qineng P. The preparation of silybinphospholipid complex and the study on its pharmacokinetics in rats. Int J Pharm. 2006;307(1):77-82.

20. Maiti K, Mukherjee K, Gantait A, Saha BP, Mukherjee PK. Curcuminphospholipid complex: Preparation, therapeutic evaluation and pharmacokinetic study in rats. Int J Pharm. 2007;330(1-2):155-163.

21. Maiti K, Mukherjee K, Gantait A, Saha BP, Mukherjee PK. Enhanced therapeutic potential of naringenin-phospholipid complex in rats. J Pharm Pharmacol. 2006;58(9):1227-1233.

22. Jain S, Valvi PU, Swarnakar NK, Thanki K. Gelatin coated hybrid lipid nanoparticles for oral delivery of amphotericin B. Mol Pharm. 2012;9(9):2542-2553.

23. Ruan LP, Chen S, Yu BY, Zhu DN, Cordell GA, Qiu SX. Prediction of human absorption of natural compounds by the non-everted rat intestinal sac model. Eur J Med Chem. 2006;41(5):605-610.

24. Bothiraja C, Pawar AP, Dama GY, Joshi PP, Shaikh KS. Novel solventfree gelucire extract of Plumbago zeylanica using non-everted rat intestinal sac method for improved therapeutic efficacy of plumbagin. J Pharmacol Toxicol Methods. 2012;66(1):35-42.

25. Shishu, Kamalpreet, Maheshwari M. Development and evaluation of novel microemulsion based oral formulations of 5-fluorouracil using non-everted rat intestine sac model. Drug Dev Ind Pharm. 2012;38(3):294-300.

26. European Pharmacopeia, 5th ed. 1452-1453.

27. Sweetman SC. Martindale: The Complete Drug Reference, 36th ed. London: Pharmaceutical Press; 2009.

28. Bombardelli E, Patri GF. Complex compounds of bioflavonoids wit phospholipids:their preparation and use and pharmaceutical and comsmotics compositions containing them, United states patents, patent no 5,043,323(1991).

29. Grit M, Crommelin DJ. Chemical stability of liposomes: implications for their physical stability. Chem Phys Lipids. 1993;64(1-3):3-18.

30. Teagarden DL, Baker DS. Practical aspects of lyophilization using non-aqueous co-solvent systems. Eur J Pharm Sci. 2002;15(2): $115-133$.

31. Elgindy N, Elkhodairy K, Molokhia A, Elzoghby A. Lyophilization monophase solution technique for improvement of the physicochemical properties of an anticancer drug, flutamide. Eur J Pharm Biopharm. 2010;74(2):397-405. 
32. Salazar J, Heinzerling O, Müller RH, Möschwitzer JP. Process optimization of a novel production method for nanosuspensions using design of experiments (DoE). Int J Pharm. 2011;420(2):395-403.

33. Elsheikh MA, Elnaggar YS, Gohar EY, Abdallah OY. Nanoemulsion liquid preconcentrates for raloxifene hydrochloride: optimization and in vivo appraisal. Int J Nanomedicine. 2012;7:3787-3802.

34. Elnaggar YS, El-Massik MA, Abdallah OY. Self-nanoemulsifying drug delivery systems of tamoxifen citrate: design and optimization. Int $J$ Pharm. 2009;380(1-2):133-141.

35. Xu K, Liu B, Ma Y, et al. Physicochemical properties and antioxidant activities of luteolin-phospholipid complex. Molecules. 2009;14(9): 3486-3493.

36. Abdelwahed W, Degobert G, Stainmesse S, Fessi H. Freeze-drying of nanoparticles: formulation, process and storage considerations. Adv Drug Deliv Rev. 2006;58(15):1688-1713.

37. Doro PL. Development of Validation of Analytical Methodology for Determination of Content and Dissolution Evaluation [master thesis]. Catholic University of Goias, Portugal; 2009.

38. Ghazal HS, Dyas AM, Ford JL, Hutcheon GA. In vitro evaluation of the dissolution behaviour of itraconazole in bio-relevant media. Int $J$ Pharm. 2009;366(1-2):117-123.

39. Lennernäs H, Nylander S, Ungell AL. Jejunal permeability: a comparison between the ussing chamber technique and the single-pass perfusion in humans. Pharm Res. 1997;14(5):667-671.

40. Sharma P, Chawla HP, Panchagnula R. LC determination of cephalosporins in in vitro rat intestinal sac absorption model. J Pharm Biomed Anal. 2002;27(1-2):39-50.
41. Barthe L, Bessouet M, Woodley JF, Houin G. The improved everted gut sac: a simple method to study intestinal P-glycoprtoein. Int J Pharm. 1998; 173:255-258.

42. Kaul S, Ritschel WA. Studies of the intestinal transfer of coumarin and 7-hydroxycoumarin across guinea pig and rat small intestine. Arzneimittelforschung. 1981;31(5):790-795.

43. Genty M, González G, Clere C, Desangle-Gouty V, Legendre JY. Determination of the passive absorption through the rat intestine using chromatographic indices and molar volume. Eur J Pharm Sci. 2001;12(3):223-229.

44. Dixit P, Jain DK, Dumbwani J. Standardization of an ex vivo method for determination of intestinal permeability of drugs using everted rat intestine apparatus. J Pharmacol Toxicol Methods. 2012;65(1):13-17.

45. Balimane PV, Chong S, Morrison RA. Current methodologies used for evaluation of intestinal permeability and absorption. J Pharmacol Toxicol Methods. 2000;44(1):301-312.

46. Le Ferrec $\mathrm{E}$, Chesne $\mathrm{C}$, Artusson $\mathrm{P}$, et al. In vitro models of the intestinal barrier. The report and recommendations of ECVAM Workshop 46. European Centre for the Validation of Alternative methods. Altern Lab Anim. 2001;29(6):649-668.

47. Gandia P, Lacombe O, Woodley J, Houin G. The perfused everted intestinal segment of rat. 1st communication: absorption kinetics of markers of different permeation mechanisms. Arzneimittelforschung. 2004;54(8):467-473.
International Journal of Nanomedicine

\section{Publish your work in this journal}

The International Journal of Nanomedicine is an international, peerreviewed journal focusing on the application of nanotechnology in diagnostics, therapeutics, and drug delivery systems throughout the biomedical field. This journal is indexed on PubMed Central,

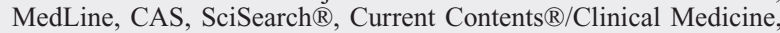

\section{Dovepress}

Journal Citation Reports/Science Edition, EMBase, Scopus and the Elsevier Bibliographic databases. The manuscript management system is completely online and includes a very quick and fair peer-review system, which is all easy to use. Visit http://www.dovepress.com/ testimonials.php to read real quotes from published authors. 